INRA Prod. Anim.,

2014, 27 (2), 161-174

\title{
Changements et innovations dans les systèmes d'élevage en Afrique
}

\author{
E. VALL, P. SALGADO, C. CORNIAUX, M. BLANCHARD, C. DUTILLY, V. ALARY \\ CIRAD, UMR112 Selmet, Campus International de Baillarguet, F-34398 Montpellier, France \\ INRA, UMR868 Selmet, 2 Place Viala, F-34060 Montpellier, France \\ Montpellier SupAgro, UMR868 Selmet, 2 Place Viala, F-34060 Montpellier, France \\ Courriel : eric.vall@cirad.fr
}

\begin{abstract}
L'Afrique, souvent qualifiée de géant endormi quand on évoque la modernisation de son agriculture, présente en réalité des dynamiques d'innovations diversifiées en particulier dans les systèmes d'élevage, qu'il est intéressant de mettre en perspective avec les changements locaux et globaux et les évolutions de l'élevage.
\end{abstract}

En Afrique, l'élevage occupe une place importante sur le plan humain, économique et environnemental (encadré 1). Il fait face à une évolution importante de la demande et il est en proie à de multiples changements (Steinfeld et al 2006, FAO 2009). L'élevage du futur devra être à la fois plus productif et plus respectueux de l'environnement et pour cela les éleveurs devront innover (Coulon et al 2011).

Au cours des dernières décennies, l'élevage africain a subi et a contribué à de multiples changements (Steinfeld et al 2006, FAO 2009, Robinson et al 2011). Les causes des changements furent d'abord principalement locales (accroissement de la population par exemple...) ; elles ont progressivement monté en globalité (ainsi de la mondialisation des échanges...) ; enfin, aujourd'hui, et de plus en plus, les effets des changements globaux se manifestent en retour sur les localités de l'élevage (cas du réchauffement climatique...). Les effets des changements sur l'élevage ont été multiples et parfois opposés, ce qui n'est pas étonnant si l'on considère des changements de nature différente, mais ce qui l'est davantage si l'on considère un même type de changement. Ainsi, par exemple, la croissance de la population a généré d'une part, de nouvelles opportunités si l'on considère l'effet de la croissance urbaine sur l'augmentation de la demande en produits animaux tant quantitative que qualitative, mais aussi d'autre part, de nouvelles contraintes si l'on considère le croît de la population rurale et l'extension des surfaces qu'il a induit, entraînant une réduction des parcours et plus d'entraves à la mobilité des troupeaux. Cet exemple montre que la question des changements ayant affecté l'éle- vage localement et globalement au cours des dernières décennies n'est pas simple et méritait un essai de synthèse, premier objectif de l'article.

Malgré la permanence de certains traits dominants, comme son caractère familial traditionnel, peu intensif (faible recours aux intrants et capital limité) et peu productif (mises bas espacées, faible productivité numérique et laitière) - marque d'une adaptation à un environnement écologique et économique changeant et incertain, de soins de santé médiocres, d'une pénurie alimentaire fréquente, et du manque d'infrastructures (routes, chaînes du froid...) -, face aux multiples changements l'élevage africain n'est pas resté le même (encadré 1). Les principales évolutions concernent l'augmentation du cheptel $(+124$ millions de bovins entre 1961 et 2010 selon Faostat), la diversification des systèmes d'élevage (Robinson et al 2011), l'intégration progressive au marché de ces derniers (FAO 2009), l'éradication des grandes épizooties (Lancelot et al 2011). La question des évolutions possibles des systèmes d'élevage en Afrique, en lien avec les principaux changements locaux et globaux, méritait aussi un essai de synthèse et de schématisation afin de dégager quelques éléments de prospective, deuxième objectif de l'article.

Ces évolutions ne se sont pas réalisées sans innovation, c'est-à-dire sans changements de pratiques au sens large se diffusant au sein des communautés des acteurs de l'élevage. En Afrique, les innovations ont été moins spectaculaires que dans d'autres régions émergentes du Sud, probablement en raison d'effets de verrouillage des systèmes d'élevage plus forts du fait de l'aléa climatique, de leur dépendance à des écosystèmes fragiles, des nombreuses incertitudes économiques, de l'insuffisance chronique du conseil et des politiques publiques. Les innovations se sont donc faites davantage à la marge, par petite touche, progressivement et avec prudence. Elles sont donc plus difficiles à déceler, et un des rôles de la recherche consiste donc à les identifier, à les évaluer et à accompagner leur développement. A la fois techniques et organisationnelles, elles ont néanmoins induit des perturbations à tous les niveaux (technique, économique, environnemental, social, politique...) touchant les domaines de la ressource, du foncier et du territoire, des systèmes de production, des filières, des réglementations, etc. Par exemple, l'adoption de la traction animale en Afrique de l'Ouest (Vall et al 2003) en augmentant l'énergie disponible sur les exploitations agricoles, a induit une forte extension des cultures ce qui a réduit les parcours et remis en cause les règles de vaine pâture, a facilité les flux de matière organique dans les terroirs et la mise en marché des produits grâce au transport, a sécurisé l'économie des ménages par le développement d'une épargne sur pied, a permis le développement de services locaux (forgerons, vétérinaires...) et a nécessité des actions politiques (vulgarisation et conseil, crédits...). Pour comprendre la demande en termes d'innovations dans un environnement en proie à de multiples changements, il faut non seulement considérer les causes des dynamiques de l'élevage induites par les changements, mais aussi explorer conjointement les effets multiples des évolutions aux différentes échelles (notamment à l'échelle des filières et des territoires, deux vecteurs privilégiés de l'innovation; Poccard et al 2011) 
afin d'assurer l'adaptation de l'élevage, et encore impliquer davantage les éleveurs dans les processus de conception des innovations de façon à mieux prendre en compte leurs objectifs et leurs contraintes. C'est le champ de la conception de systèmes d'élevage innovants, qui peut prendre différentes formes en fonction de l'importance du changement visé (adaptation, conception pas à pas, conception de novo; Dedieu et al 2011, Meynard et al 2012) et du degré de participation des éleveurs (co-conception ; Vall et Chia 2014). Comme il est difficile de présenter un panorama général et synthétique du spectre des innovations en cours, nous avons sélectionné des innovations illustrant ce que les acteurs font pour lever les principales contraintes liés aux changements de contexte et aux évolutions de l'élevage (problèmes d'accès aux ressources et de mobilité, problème pour accroitre la productivité à moindre coût, problème pour améliorer l'intégration au marché), ce qui constitue le troisième objectif de l'article.

Les changements génèrent des opportunités et des contraintes pour les éleveurs, et ces derniers innovent pour s'adapter et anticiper le futur, ce qui induit des évolutions concernant les systèmes d'élevage, dont certains effets agissent en retour sur les changements. Après un bref rappel de la diversité des systèmes d'élevage en Afrique (partie 1), l'article propose une analyse de cette chaine de causalité en abordant les questions suivantes : Comment les changements locaux et globaux, passés et récents affectent-ils les systèmes d'élevage (partie 2) ? Face aux opportunités et aux contraintes générées par ces changements, quelles ont été les réactions des éleveurs et comment ont-ils innovés (partie 3) ? Quelles sont les conséquences des innovations et des diverses dynamiques impulsées directement par les changements sur les évolutions des systèmes d'élevage, et enfin quel est l'impact de ces évolutions en terme d'atténuation ou d'aggravation des changements (partie 4) ?

\section{Encadré 1. L'importance de l'élevage en Afrique.}

En 2010, la population agricole africaine (estimée à 560 millions d'habitants) dépendait en grande partie de l'élevage de 312 millions de grands ruminants (dont $92 \%$ de bovins), 642 millions de petits ruminants (à parts égales d'ovins et de caprins), 25 millions d'équidés (dont $73 \%$ d'ânes) et de 1,8 milliards de monogastriques (98\% de volailles et $2 \%$ de porcins). Les pâturages représentent une part très importante de la surface agricole $(78 \%)$, mais sont de valeurs très inégales selon la zone agro-écologique.

Sources : Faostat et Robinson et al (2011).

\begin{tabular}{|c|c|c|}
\hline Superficie (en millions de $\mathbf{k m}^{2}$ ) & 1961 & 2010 \\
\hline Afrique & 30,3 & 30,3 \\
\hline Eaux intérieures & 0,7 & 0,7 \\
\hline Espaces terrestres & 29,6 & 29,6 \\
\hline Déserts & $10,7^{(1)}$ & $11,2^{(1)}$ \\
\hline Forêts & $8,4^{(1)}$ & $6,7^{(1)}$ \\
\hline Surface agricole : & 10,6 & 11,7 \\
\hline Cultures annuelles & 1,5 & 2,3 \\
\hline Cultures permanentes & 0,1 & 0,3 \\
\hline Pâturages & 8,9 & 9,1 \\
\hline \multicolumn{3}{|l|}{ Cheptel (en milliers de têtes) } \\
\hline Camélidés & 8626 & 22398 \\
\hline Bovins & 122537 & 285527 \\
\hline Buffles & 1501 & 3818 \\
\hline Ovins & 135126 & 311764 \\
\hline Caprins & 94255 & 330514 \\
\hline Chevaux & 3494 & 6103 \\
\hline Anes & 10984 & 18793 \\
\hline Mulets & 1837 & 705 \\
\hline Porcins & 5668 & 31440 \\
\hline $\begin{array}{l}\text { Systèmes d'élevage d'Afrique } \\
\text { subsaharienne en } 2000\end{array}$ & $\begin{array}{l}\text { Espace occupé } \\
\text { (millions de } \mathrm{km}^{2} \text { ) }\end{array}$ & $\begin{array}{l}\text { Effectif de bovins } \\
\text { (millions UBT }^{(2)} \text { ) }\end{array}$ \\
\hline Systèmes pastoraux & 13,4 & 73,4 \\
\hline Systèmes mixtes & 6,6 & 134,4 \\
\hline Systèmes spécialisés & 4,1 & 13,6 \\
\hline Total & 24,1 & 221,4 \\
\hline
\end{tabular}

(1) estimations des auteurs à partir des données Faostat

(2) 1 UBT = 1 bovin de $250 \mathrm{~kg}$

L'élevage occupe une part significative dans l'économie des pays africains dont il représente en moyenne $15 \%$ du PIB. Ce ratio s'élève à plus de $40 \%$ dans certaines régions d'élevage comme dans les pays sahéliens d'Afrique de l'Ouest (Club du Sahel 2008). II contribue significativement à la sécurité alimentaire, à la réduction de la pauvreté et génère des emplois (FAO 2009). II est également une importante source d'énergie agricole (Lhoste et al 2010), un pourvoyeur de matière organique pour fertiliser les sols (Bouwman et Van der Hoeck 1997) et représente la principale forme d'épargne des ménages ruraux qui ont peu accès au système bancaire (Alary et al 2011). En outre, les échanges non marchands de bétail sont un facteur essentiel d'intégration et de cohésion sociale (Faye et Alary 2001). 


\section{1 / Diversité des systèmes d'élevage en Afrique}

En Afrique, les élevages d'herbivores dépendent largement de prélèvements directs sur la végétation spontanée et cultivée si bien que la carte des systèmes d'élevage épouse grosso modo la répartition spatiale de la végétation, qui est elle même déterminée par les régimes pluviométriques et par les températures. Cette cartographie sommaire peut être nuancée si l'on prend en compte l'altitude, la présence de vecteurs de maladies (comme les glossines), la proximité d'un cours d'eau et de périmètres irrigués (Nil, Niger...), la présence de grands centres urbains ou au contraire l'enclavement. C'est ainsi que les systèmes pastoraux dominent dans les zones arides ou subhumides peu peuplées, que les systèmes mixtes avec intégration de l'agriculture pluviale et de l'élevage dominent dans les zones plus densément peuplées en zone de savanes subsaharienne, en zones à climat méditerranéen en Afrique du Nord et du Sud et sur les hautes terres (Ethiopie, Kenya, Madagascar...), que les systèmes mixtes associés aux cultures irriguées dominent dans les zones de delta (Nil, Niger...), et que les systèmes spécialisés se multiplient dans les zones périurbaines (Alary et Lhoste 2002, Robinson et al 2011 ; figure 1 ).

Aujourd'hui, les systèmes mixtes sont numériquement majoritaires en Afrique (photo 1). Selon les données de Robinson et al (2011), ils concernent environ 60\% du cheptel bovin contre $34 \%$ pour les systèmes pastoraux (encadré 1). Cette évolution est récente puisqu'en 2000 Boutonnet et al (2001) estimaient que les systèmes pastoraux couvraient environ $60 \%$ de l'offre en viande bovine, de petits ruminants et de lait. Les systèmes mixtes occupent moins d'espace que les systèmes pastoraux, puisqu'ils ne concernent, en moyenne, que $24 \%$ de la surface des pays contre $62 \%$ pour les systèmes pastoraux (Robinson et al 2011 ; encadré 1). En conséquence, ils ont un caractère plus intensif et dans les régions où ils se développent, la densité moyenne de bovins s'élève à environ 9,9 têtes $/ \mathrm{km}^{2}$ contre 2,2 têtes $/ \mathrm{km}^{2}$ dans les zones pastorales. Enfin, les régions concernées par les systèmes mixtes concentrent $68 \%$ de la population du continent, ce qui les place en contact direct avec un marché potentiel très important. Du fait de leur importance numérique qui ne fait que croître, ils constituent un levier important pour le développement de l'élevage en Afrique (Herrero et al 2010).

Les systèmes mixtes d'agriculture et d'élevage dominent largement dans les
Figure 1. Répartition spatiale des principaux systèmes d'élevage en Afrique (carte adaptée de Robinson et al 2011).

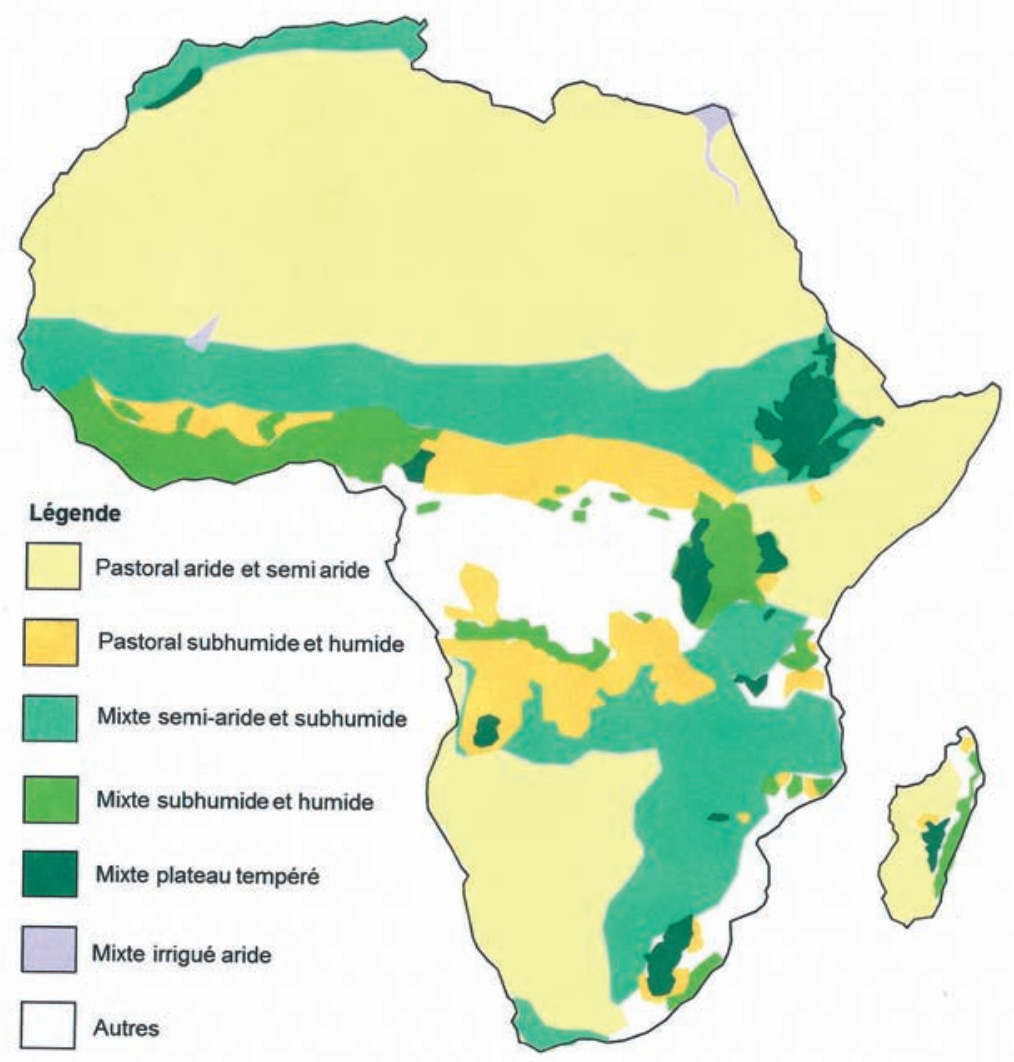

zones semi-arides à subhumides et sur les hautes terres où une pluviométrie plus favorable (>500 mm/an environ) rend possible la diversification des cultures et des élevages (herbivores et monogastriques). Ils sont également présents dans les zones humides, où la présence de la trypanosomose conduit à privilégier l'élevage de taurins trypanotolérants et de monogastriques ainsi

que dans les zones de delta où ils sont associés à des systèmes de cultures hautement productifs en biomasses végétales (riz, maïs...) dont l'élevage profite. Dans les zones où la densité de population est élevée et en augmentation $\left(>40 \mathrm{hab} . / \mathrm{km}^{2}\right)$, la pression humaine sur les terres rend nécessaire des formes d'association de l'agriculture et de l'élevage qui produisent des effets d'intensification

Photo 1. Système mixte de polyculture-élevage (épandage de fumure organique et culture attelée dans des rizières à Madagascar; cliché M. Blanchard).

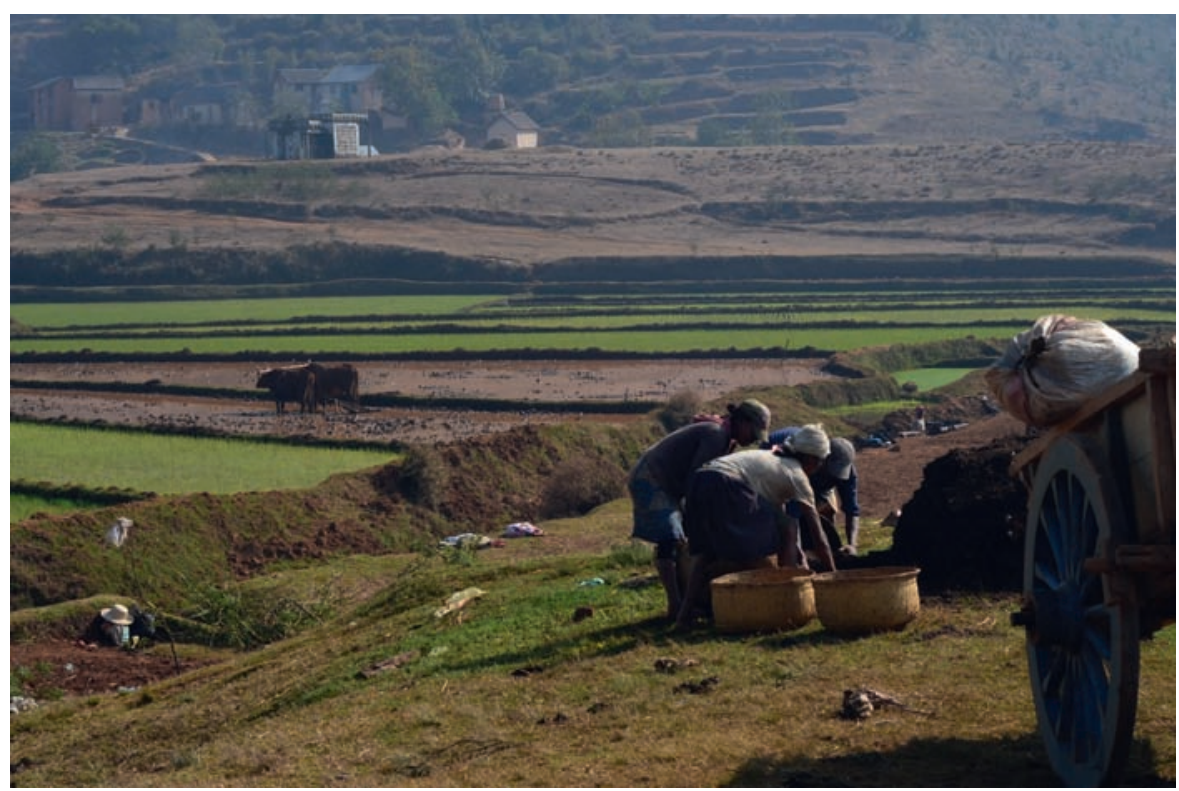


Photo 2. Troupeau ovin sur une steppe aride dans l'oriental marocain ; cliché M. Acherkouk.

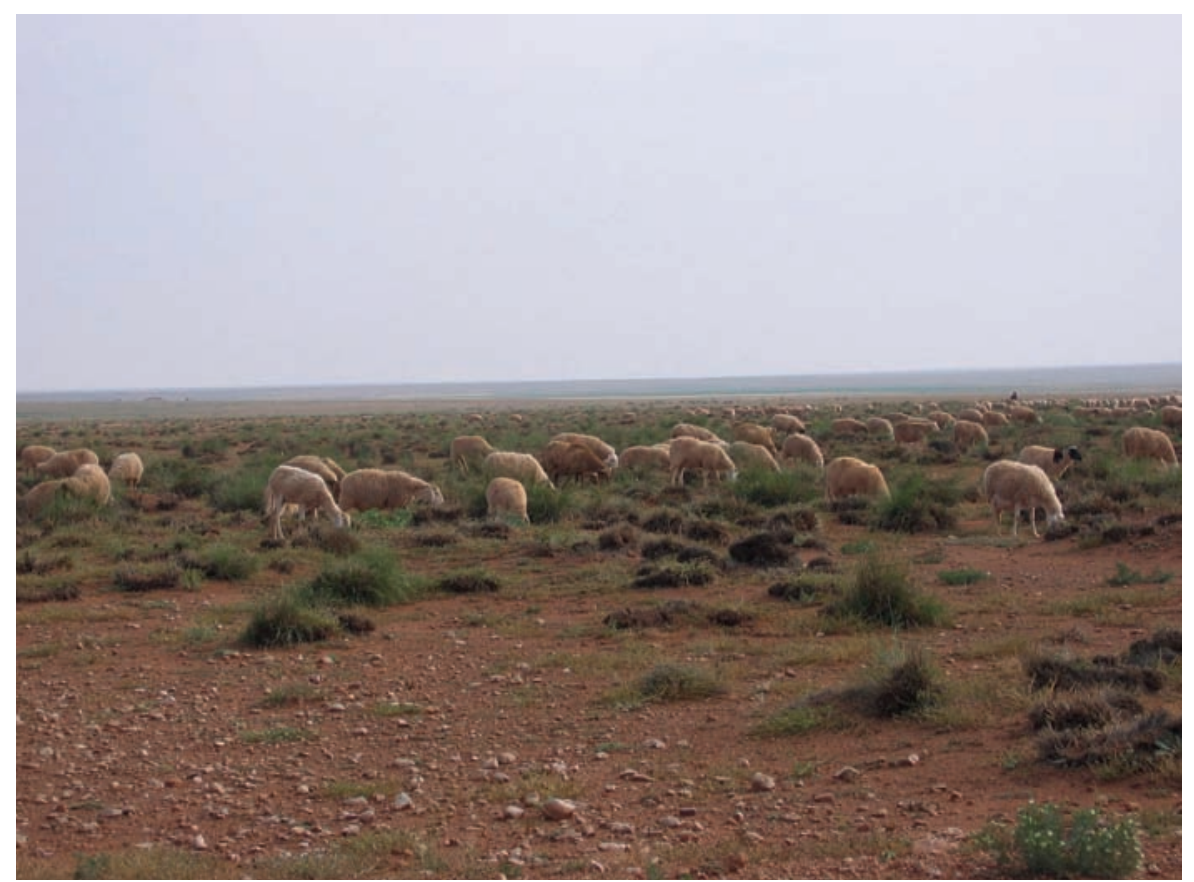

Photo 3. Système péri-urbain en voie d'intensification (élevage laitier proche de Sikasso au Mali ; cliché E. Vall)

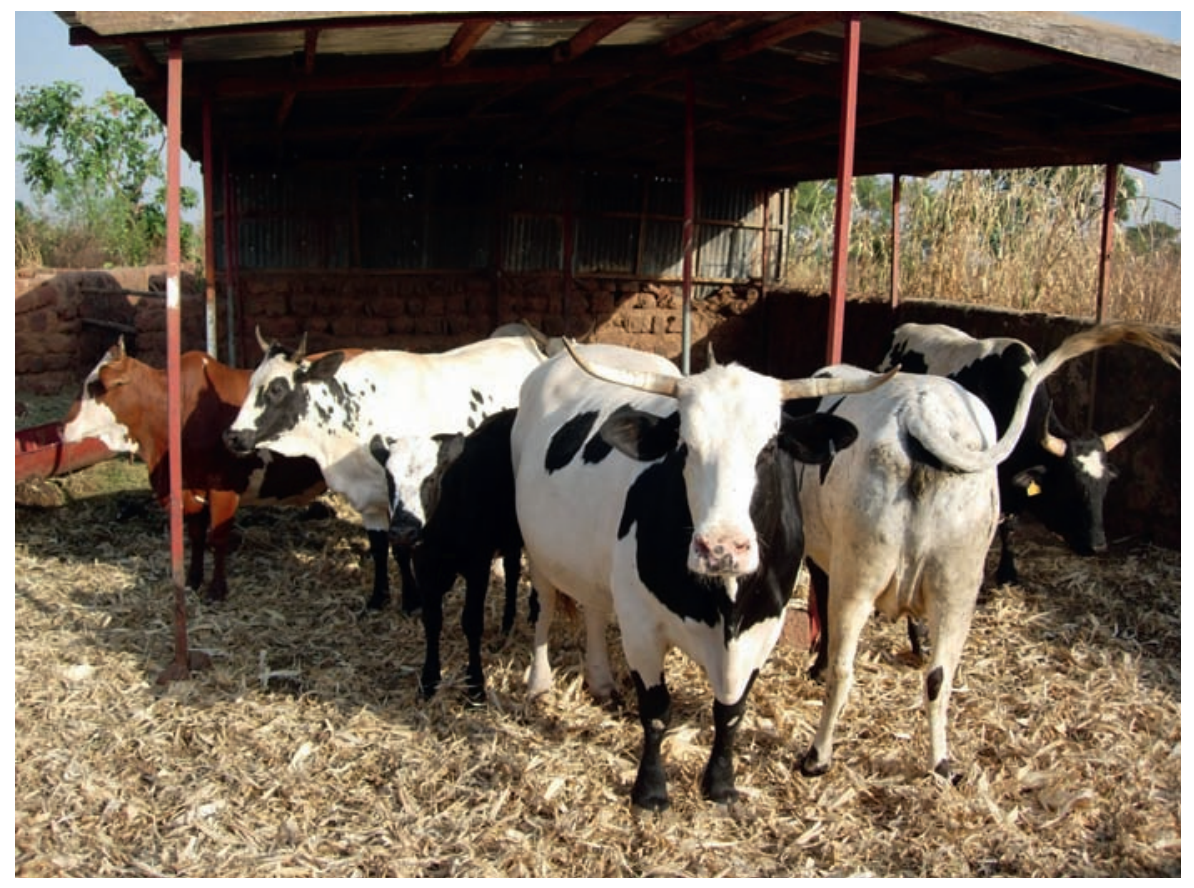

de la production. Les bovins et équins fournissent de l'énergie pour les travaux de culture et les transports ; ils bénéficient d'apports alimentaires de l'espace cultivé (résidus de culture, cultures fourragères, sous-produits agro-industriels...) et restituent des éléments fertilisants aux terres cultivées (compost, lisier, fumier...). S'ajoutent à ces flux des complémentarités économiques entre culture et élevage (épargne sur pied des revenus agricoles...), la création d'emploi (bergers, forgerons...), des effets d'intégration sociale entre agri- souvent inférieure à $10 \mathrm{hab} . / \mathrm{km}^{2}$ et se concentrent principalement dans les zones semi-arides et arides. Ils sont reconnus pour être la principale forme de valorisation à la fois économique et écologique de ces milieux secs (photo 2). On les retrouve dans les zones de savanes humides et subhumides faiblement peuplées situées de part et d'autre de l'équateur et dans les zones semi-arides à arides méditerranéennes. Ils se caractérisent par une forte mobilité des troupeaux d'herbivores domestiques (bovins, ovins, caprins) et un recours important aux parcours naturels ouverts («les communs »). La mobilité des troupeaux est une condition nécessaire à la viabilité de ces systèmes, pour accéder à des ressources en eau et en pâturage dont la répartition spatiale varie au cours de l'année. Mais, aujourd'hui, elle est de plus en plus contrainte par l'extension des cultures dans les zones semi-arides et surtout dans les régions subhumides qui étaient traditionnellement fréquentées par les grandes transhumances, mais aussi du fait de la dégradation des parcours dans les zones arides et semiarides méditerranéennes. La part de l'élevage dans le revenu et l'autoconsommation du ménage reste élevée. Ces systèmes se trouvent le plus souvent dans des zones enclavées, peu sécurisées, et ils sont contraints par l'aridité du climat ou par une pression parasitaire importante dans les zones subhumides. Ils représentent les formes d'élevage les plus anciennes d'Afrique, mais aujourd'hui, sous l'effet de l'extension des zones agricoles et avec la raréfaction des zones de parcours, leur aire d'extension tend à se circonscrire aux zones où l'agriculture est très contrainte voire impossible. Des conversions vers le système agro-pastoral s'observent parfois quand l'agriculture est possible. L'aspiration des pasteurs à bénéficier de conditions de vie plus confortables, quand elle ne peut être satisfaite, conduit parfois à certaines formes de crise du pastoralisme (Bourbouze 2007).

Les systèmes périurbains connaissent un développement rapide en réponse à la croissance de la demande des villes en produits animaux. Ils concernent souvent des espèces à cycles courts (volaille, porcs) et des formes intensifiées d'élevage de ruminants (embouche, lait ; photo 3). Ils forment en réalité un ensemble assez hétérogène d'élevages si l'on considère leur capital d'exploitation allant des petits élevages familiaux urbains et périurbains sans gros investissement et fonctionnant sur le marché de proximité, aux élevages souvent dirigés par des citadins, parfois par de gros fermiers installés depuis plusieurs générations, comme en Afrique australe, disposant d'une solide capacité d'investissement, d'un titre foncier, de 
bâtiments (logement animaux en dur, fenil, clôture...) et d'équipements (motorisation, chaine du froid...). L'espace disponible étant limité en périphérie des villes, l'intensification est clairement la logique poursuivie avec un recours important aux intrants (cultures fourragères, aliments bétail, insémination artificielle...), à des salariés et à des équipements modernes (Duteurtre 2007, Daburon 2013). Ce confinement en milieu urbain est parfois la source de pollution et de nuisances liées au devenir des effluents d'élevage. Une fraction importante de ces nouveaux éleveurs est inexpérimentée et peu conseillée, et dans ce cas la viabilité de l'élevage est compromise (surdimensionnement des investissements, mauvaise valorisation des rations, problèmes sanitaires...). Un service d'accompagnement stratégique et technique constituerait une priorité pour ce type d'élevage, afin de les aider à mener à bien leur intensification, à répondre à la demande des consommateurs urbains, à satisfaire aux exigences sanitaires et environnementales des collectivités et des populations riveraines, mais avec le désengagement des Etats cet appui conseil a du mal à émerger (Faure et al 2014).

Les systèmes d'élevage industriels, dits parfois " hors sols », sont encore peu représentés sur le continent, et sont pour l'instant principalement localisés en Afrique du Nord (Sraïri 2011) et en Afrique australe (cf. site web de la $\mathrm{SAFA}^{1}$ ). Ils sont généralement intégrés dans une filière, spécialisés sur un type de production et conduits dans une logique d'économie d'échelle (baisse du coût unitaire d'un produit par augmentation de la quantité de sa production). Ils se caractérisent, donc, par un niveau élevé d'intensification et de technicité, une alimentation produite en grande partie en dehors de l'exploitation, de fortes charges animales. Ils concernent le plus souvent des élevages de monogastriques comme les poules pondeuses, les poulets de chair et les porcins, mais également l'aquaculture et les élevages laitiers. Ils posent parfois des problèmes de pollution et de bienêtre animal. Ils représentent une sorte d'aboutissement de la logique d'intensification " industrielle » et d'économie d'échelle, déjà à l'œuvre dans les systèmes périurbains et marquent l'abandon des complémentarités de productions évoquées dans les autres systèmes. Les législations en vigueur n'accordent encore que peu d'importance aux aspects environnementaux, ce qui a permis jusqu'ici d'assurer des conditions favorables à l'essor relatif de ces élevages intensifiés. Mais l'émergence de ces questions imposera certainement une révision de leurs conditions de travail.

\section{2 / L'engrenage des chan- gements et leur impact sur l'élevage}

Depuis 1960, période de la décolonisation, l'Afrique a été le théâtre de nombreux changements entraînant des impacts multiples sur l'élevage, du niveau local au niveau le plus global. Nous allons décrire leur nature et leurs conséquences en spécifiant les interactions éventuelles entre changements, et selon les niveaux d'échelle (figure 2).

\section{1 / Les conséquences de la crois- sance démographique}

La croissance démographique a sans doute été, et reste, le changement le plus déterminant sur le développement de l'élevage par ses multiples effets directs et indirects. D'environ 280 millions d'habitants en 1960, la population africaine est passée à un peu plus d'un milliard en 2010, soit un croît d'environ $2,5 \%$ /an (contre 1,6\%/an au niveau mondial), et elle continuera à augmenter pour se situer entre 1,9 et 2,5 milliards en 2050 (United Nations 2010).

Cette croissance a induit une forte densification de l'espace rural, qui est passée de 8 à 35 hab. $/ \mathrm{km}^{2}$ entre 1960 et 2010 [(1) sur la figure 2]. Durant cette période, la production agricole a augmenté principalement par accroissement du cheptel et par extension des surfaces cultivées, la faible densité de population initiale ayant favorisé une telle évolution (Dorin et al 2011, Petit 2011). L'élevage a le plus souvent conservé un caractère traditionnel, une forte dépendance directe aux ressources naturelles et une faible productivité. Entre 1960 et 2010, le cheptel bovin a doublé passant de 123 à 246 millions de têtes, alors que l'indice de production par tête n'a progressé que de $1 \%$ contre plus de $120 \%$ en Asie du SudEst (Faostat $\left.{ }^{2}\right)$.

Mais cette stratégie de croissance atteint progressivement des limites car les terres disponibles se raréfient [(2) sur la figure 2]. Dans les zones agropastorales, les parcours communs dévolus à l'élevage sont peu à peu remplacés par des cultures. La compétition sur les terres disponibles est de plus en plus forte. Les pouvoirs coutumiers montrent des limites pour gérer l'accès aux res- sources, si bien qu'entre les nouveaux usagers les conflits augmentent. La mobilité des troupeaux sur les territoires est réduite en période de culture ce qui pose des problèmes d'alimentation à des périodes où cela ne se posait pas avant (Bourbouze 2007, Vall et Diallo 2009). Dans les zones subhumides de l'Afrique de l'Ouest, le syncrétisme culturel a longtemps favorisé la coexistence pacifique de populations de cultures différentes, composées d'agriculteurs autochtones et d'éleveurs quittant le Sahel et ses sécheresses à répétition. Mais aujourd'hui les compétitions sur le foncier et les ressources se sont durcies, les conflits liés à l'utilisation des terres se multiplient et se cristallisent sur des clivages motivés aussi par des sentiments d'appartenance ethnique ou religieuse.

Un autre aspect de la croissance démographique africaine concerne l'accélération de l'urbanisation. En 1960 deux villes dépassaient le million d'habitants contre 57 aujourd'hui. Entre 1960 et 2010 la proportion de la population urbaine africaine est passée de 19 à $60 \%$ (Faostat). Un croît naturel de la population élevé conjugué aux migrations de ruraux vers les villes ont soutenu ce mouvement [(3) sur la figure 2]. La demande en produit animaux s'en trouve quantitativement augmentée, d'autant plus que les classes moyennes et aisées ont plus d'exigence sur la qualité. Des filières se mettent en place dans les ceintures périurbaines pour répondre à la demande locale, ou bien à l'échelle régionale pour alimenter les mégapoles situées généralement loin des zones traditionnelles d'élevage (Boutonnet et al 2001, FAO 2009). La production africaine a du mal à satisfaire la croissance de la demande. Dans la plupart des zones de l'Afrique subsaharienne, un déficit important en viande et en lait est avéré (Boutonnet et al 2001). Une part importante de la demande est couverte par les importations extraafricaines (poulets congelés, lait en poudre...) notamment dans les pays côtiers disposant d'installations portuaires.

L'attrait de la ville est fort chez les enfants des éleveurs. Les jeunes aspirent à plus d'autonomie et l'on remarque qu'au sein des familles les réseaux de parentèle se recomposent et tendent à se restreindre. Avec la modernisation des campagnes, le mode de vie fondé sur la mobilité est progressivement remis en cause, quand bien même il s'agit d'une mobilité à temps partiel, parce qu'elle complique l'accès à l'école, aux services de santé, la participation au développement local, etc. (Dongmo et al 2007).

\footnotetext{
1 South African Feedlot association http://www.safeedlot.co.za

2 http://faostat.fao.org/
} 
Figure 2. Les changements ayant affecté l'élevage en Afrique de 1960 à aujourd'hui, du local au global.

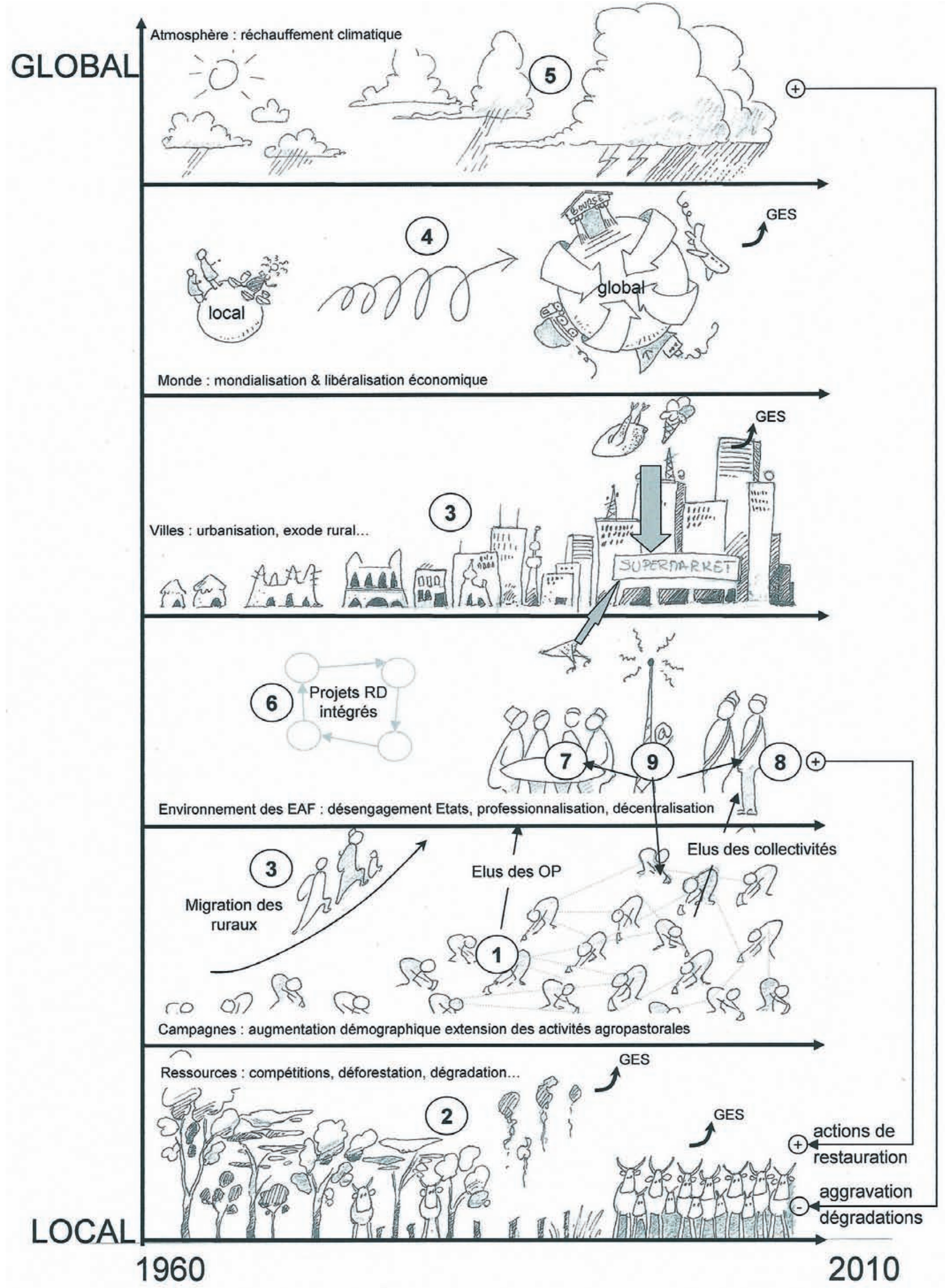


Tableau 1. Evolution des quantités de produits animaux produites, importées et consommées selon les continents entre 1960 et 2010 (d'après Faostat).

\begin{tabular}{|c|c|c|c|c|c|c|c|c|c|}
\hline & & \multicolumn{2}{|c|}{$\begin{array}{l}\text { Production } \\
\text { (kg/an/hab.) }\end{array}$} & \multicolumn{2}{|c|}{$\begin{array}{l}\text { Importation } \\
\text { (kg/an/hab.) }\end{array}$} & \multicolumn{2}{|c|}{$\begin{array}{l}\text { Consommation } \\
\text { (kg/an/hab.) }^{(1)}\end{array}$} & \multicolumn{2}{|c|}{$\begin{array}{l}\text { Coefficient d'augmentation } \\
\text { entre } 1961 \text { et } 2010\end{array}$} \\
\hline & & 1961 & 2010 & 1961 & 2010 & 1961 & 2010 & $\begin{array}{c}\text { Prod. Totale } \\
\text { Ian }\end{array}$ & $\begin{array}{l}\text { Conso. } \\
\text { lan/hab. }\end{array}$ \\
\hline \multirow{5}{*}{$\begin{array}{l}\text { Viande } \\
\text { bovine }\end{array}$} & Afrique & 7,1 & 6,2 & 0,4 & 0,4 & 6,8 & 6,4 & 2,9 & 0,9 \\
\hline & Asie & 1,6 & 4,1 & 0,0 & 0,7 & 1,6 & 4,2 & 6,1 & 2,7 \\
\hline & Amérique du Sud & 30,9 & 38,0 & 0,1 & 0,9 & 27,3 & 31,3 & 3,2 & 1,1 \\
\hline & Amérique du Nord & 38,9 & 39,1 & 2,0 & 3,5 & 40,7 & 38,9 & 1,6 & 1,0 \\
\hline & Europe & 15,0 & 14,9 & 1,4 & 5,4 & 15,5 & 16,1 & 1,1 & 1,0 \\
\hline \multirow{5}{*}{ Volaille } & Afrique & 1,3 & 5,2 & 0,0 & 1,3 & 1,3 & 5,5 & 13,3 & 4,3 \\
\hline & Asie & 0,9 & 8,7 & 0,0 & 1,5 & 0,9 & 8,8 & 23,4 & 9,9 \\
\hline & Amérique du Sud & 2,1 & 44,2 & 0,0 & 0,8 & 2,1 & 30,2 & 54,2 & 14,4 \\
\hline & Amérique du Nord & 17,1 & 61,0 & 0,0 & 0,9 & 16,2 & 47,9 & 5,8 & 3,0 \\
\hline & Europe & 4,5 & 21,9 & 0,4 & 6,6 & 4,6 & 21,9 & 5,5 & 4,7 \\
\hline \multirow{5}{*}{ Lait ${ }^{(2)}$} & Afrique & 39,6 & 45,8 & 2,5 & 7,9 & 32,1 & 44,0 & 3,9 & 1,4 \\
\hline & Asie & 25,6 & 65,8 & 1,3 & 6,8 & 21,9 & 54,4 & 6,2 & 2,5 \\
\hline & Amérique du Sud & 95,1 & 161,4 & 5,3 & 5,9 & 80,8 & 130,0 & 4,4 & 1,6 \\
\hline & Amérique du Nord & 314,2 & 280,7 & 2,7 & 15,1 & 263,9 & 250,5 & 1,5 & 0,9 \\
\hline & Europe & 301,4 & 289,6 & 10,8 & 71,9 & 171,0 & 219,6 & 1,1 & 1,3 \\
\hline
\end{tabular}

(1) « consommation » correspond au « disponible alimentaire » selon la définition de Faostat, soit :

Disponible alimentaire $=$ Production + Importation $-($ re) Exportation.

${ }^{(2)}$ en équivalent lait (selon la grille d'équivalence de la FAO).

\section{2 / Les conséquences des chan- gements économiques}

Depuis 1960, la production et la consommation de produits animaux (viande bovine, volaille et lait) par habitant est restée faible en Afrique comparativement à l'Europe, à l'Amérique du Nord et à l'Amérique du Sud (tableau 1). Elles sont même aujourd'hui dépassées par l'Asie pour la volaille et le lait, et sans doute bientôt pour la viande bovine. On note néanmoins une hausse sensible de la consommation par habitant entre 1960 et 2010 pour la volaille et le lait. Cette hausse est issue de la hausse de la production et, dans une moindre mesure, des importations. Pour la viande bovine et le lait, l'Afrique comme l'Amérique du Sud se caractérisent par une forte absorption de l'augmentation de la production par la croissance démographique, contrairement à l'Asie. Les données continentales du tableau 1 masquent néanmoins une grande variabilité entre sous-régions et pays africains.

D'importants changements économiques sont survenus en lien avec la mondialisation [(4) sur la figure 2]. La structure des importations a considérablement évolué (FAO 2009). Dans les années 1960 l'Afrique importait 102000 tonnes de lait (principalement concentré) et 87000 tonnes de viande bovine alors qu'en 2010 elle importait 246000 tonnes de lait (en poudre principalement), 234000 tonnes de viande bovine et surtout 1066000 tonnes de volailles (Faostat). En 2010 on consommait 1,5 kg/ $\mathrm{an} /$ hab. de produits animaux importés contre $0,6 \mathrm{~kg} / \mathrm{an} / \mathrm{hab}$. dans les années 1960. De plus en plus de produits importés proviennent du continent sud-américain pour la viande (bovins, poulets du Brésil) mais aussi pour le lait (Uruguay, Argentine). En Afrique de l'Est, le commerce intra régional du bétail constitue une industrie importante en plein essor d'une valeur annuelle supérieure à 65 millions de dollars US (IIED 2009). Autre aspect de la mondialisation, l'accaparement des terres prend de l'ampleur sur le continent. Les projets d'agrobusiness (biocarburant et aliments destinés à l'export) affectent des espaces importants dans des zones peu enclavées présentant un bon potentiel agricole. Selon la Banque Mondiale, entre 2008 et 2009, 30 millions d'hectares ont été accaparés par des investisseurs. Des pays d'élevage comme Madagascar, le Soudan, l'Ethiopie et le Ghana sont particulièrement touchés puisqu'on estime qu'entre 1,0 et $2,5 \%$ des terres arables sont déjà concernées.
On observe aussi une montée de l'insécurité dans les villes et aussi dans les campagnes, liée à des causes multiples économiques, sociales et politiques. Dans les zones rurales, elle concerne particulièrement les éleveurs qui sont de plus en plus souvent rançonnés ou victimes de vol de bétail. Ce phénomène conduit certains éleveurs à restreindre les déplacements des troupeaux dans des zones peu sécurisées et à quitter les campements pour s'installer dans les cœurs de villages.

\section{3 / Les conséquences du ré- chauffement climatique}

Le réchauffement déstabilise les régimes pluviométriques, aléatoires par nature dans les régions tropicales, qui à leur tour perturbent la production primaire de biomasse et le remplissage des réserves d'eau dont l'élevage dépend directement [(5) sur la figure 2 ; Steinfeld et al 2006, FAO 2009]. En Afrique, la température annuelle de surface devrait croitre de 3 à $4^{\circ} \mathrm{C}$ pour la période 2080 2099 par rapport à son niveau de 1980 1999, avec moins de réchauffement dans les régions équatoriales et côtières (IPCC 2007). Les projections sur la pluviométrie sont moins précises. Pour la période 2080-2099, on prévoit une baisse 
de la moyenne annuelle des précipitations de l'ordre de $20 \%$ en Afrique du Nord, une augmentation de $7 \%$ en Afrique de l'Est et une baisse des pluies hivernales de $40 \%$ en Afrique australe. L'incertitude est encore plus grande concernant l'Afrique de l'Ouest (IPCC 2007). En Afrique subsaharienne, les flux nets de carbone dans l'atmosphère, qui étaient négatifs au début du $X^{\text {ème }}$ siècle, ont atteint $16,3 \%$ du total mondial en 2005. L'élevage contribue à ces flux de carbone via la fermentation entérique et par la décomposition des déjections animales. La qualité médiocre des fourrages de saison sèche réduit leur digestibilité et accroît le taux d'émission de méthane par animal (Steinfeld et al 2006). Chez des bovins nourris d'aliments présentant une digestibilité de $50 \%$ ce taux est de l'ordre de $130 \mathrm{~kg} / \mathrm{an}$, soit environ cinq fois plus que des bovins européens consommant des aliments dont la digestibilité est de 75\% (GIEC 1996).

\section{4 / Les conséquences des chan- gements de politiques de l'élevage et de santé}

A la suite du succès mitigé de la Révolution Verte en Afrique, qui a d'ailleurs peu concerné l'élevage, les politiques d'ajustements structurels mises en œuvre sous la houlette du Fond Monétaire International (FMI) et de la Banque Mondiale à partir des années 1980 ont provoqué le désengagement brusque des Etats du développement rural (Bichat 2012). Les grands projets intégrés de recherche-développement ont progressivement disparu et les services publics d'appui à l'agriculture ont vu leurs moyens humains et financiers considérablement réduits [(6) sur la figure 2]. Dans les années 1990, les acteurs privés locaux ont eu des difficultés à prendre le relais. Aujourd'hui, dans de nombreux pays les services zootechniques et vétérinaires et les services financiers ne parviennent pas à couvrir la demande. En 2008, la Banque Mondiale placait de nouveau l'investissement dans l'agriculture dans ses priorités (World Bank 2008).

Au niveau Panafricain, à l'exception du Sahel, les politiques nationales ont tendance à favoriser la sédentarisation des éleveurs. Mais, à la suite d'un processus conduit par l'Union Africaine (2006-2011), un cadre politique pour le pastoralisme a été validé en 2011 pour la promotion de l'élevage, la protection et la reconnaissance des droits des éleveurs, la création d'espaces de dialogues entre les communautés pastorales. Au niveau régional, des mesures sont prises pour faciliter la mobilité et la transhumance transfrontalière avec par exemple l'adoption d'un certificat international de transhumance mis en place par la Communauté Economique Des Etats de l'Afrique de l'Ouest (CEDEAO) en 1998. Au niveau national, des codes pastoraux existent et de nombreux accords bilatéraux encadrent les mouvements transfrontaliers. Mais, faute d'achèvement des textes et de mise en place des institutions et des personnels chargés de leur application, ces cadres politiques demeurent souvent inappliqués.

Dans le domaine de la santé (Lancelot et al 2011), d'importants moyens financiers et humains ont été consacrés à la mise au point de vaccins et de méthodes de lutte et d'éradication des vecteurs des maladies parasitaires jusque dans les années 1990. La santé du bétail s'est améliorée avec l'éradication de grandes épizooties comme la peste bovine en 2011 et avec le recul des aires infestées par les glossines (qui est aussi une conséquence indirecte positive de la déforestation). Mais de nombreuses autres maladies infectieuses et parasitaires continuent à circuler (peste des petits ruminants, péripneumonie contagieuse, pleuropneumonie contagieuse caprine, peste porcine, trypanosomiases, fièvre de la vallée du Rift...). Les mouvements d'animaux, la réduction des services de santé animale, les changements climatiques, l'urbanisation anarchique sont autant de facteurs compliquant le contrôle des maladies. En Afrique, la situation sanitaire défavorable pour les hommes et pour les animaux a été, et demeure, une cause importante de la lenteur de son développement (Bichat 2012)

\section{5 / Les conséquences de la pro- fessionnalisation des éleveurs et de la décentralisation}

Depuis les années 1990, de nombreuses organisations paysannes ont émergé [(7) sur la figure 2]. Aujourd'hui, les organisations les plus puissantes concernent l'agriculture mais des organisations d'éleveurs se structurent et fondent des réseaux régionaux. En Afrique de l'Est et Australe, principalement au Kenya, en Afrique du Sud et en Namibie, des organisations d'éleveurs nationales ont atteint un niveau de professionnalisation élevé et tiennent à jour des bases de données sur les pédigrées et les performances des différentes races locales, ce qui permet d'améliorer la productivité des troupeaux des éleveurs qui intègrent ces réseaux. En Afrique de l'Ouest et Centrale, des organisations d'éleveurs locales et nationales se sont structurées depuis plusieurs décennies. Des réseaux régionaux se sont mis en place comme l'Association pour la Promotion de l'Elevage au Sahel et en Savane (Apess) et le réseau Billital Marrobé. Ces organisations poursuivent des objectifs multiples comme le renforcement des capacités des éleveurs (alimentation et reproduction) par l'information et la formation, un accès facilité aux intrants (aliments, soins), l'organisation de la mobilité et des transhumances transfrontalières, la valorisation des produits de l'élevage, l'appui juridique ou encore la valorisation du patrimoine culturel du monde pastoral. A l'avenir ces organisations d'éleveurs pourraient jouer un rôle crucial dans la conservation des races locales (adaptées aux milieux difficiles) et pour l'amélioration génétique à travers la sélection et l'insémination artificielle. D'ailleurs, sous l'influence du marché et notamment dans les élevages intensifs, les éleveurs privilégient souvent le caractère productif et on observe une adaptation des types d'animaux (Planchenault et Boutonnet 1997, Abdelguerfi et Laouar 2000).

Les politiques de décentralisation ayant conduit ces dernières années à la mise en place de collectivités territoriales auxquelles les Etats transfèrent progressivement des fonctions de gestion des ressources naturelles, produisent localement des changements importants. Elles offrent aux éleveurs des espaces de concertation pour s'impliquer dans la gouvernance des territoires et dans la gestion des ressources pastorales locales [(8) sur la figure 2].

\section{6 / Les conséquences du déve- loppement des technologies de la communication}

Au cours des 10 dernières années, le développement du téléphone portable a réduit significativement l'isolement géographique des éleveurs (et notamment des communautés pastorales enclavées) et a profondément transformé les pratiques des éleveurs [(9) sur la figure 2]. Il permet de communiquer avec les éclaireurs et les bergers, et par conséquent de mieux organiser les déplacements des troupeaux. Il est utilisé pour organiser les poursuites des voleurs de bétail. Il permet aux commerçants du bétail d'organiser des réseaux de rabatteurs, de suivre l'offre en animaux sur le marché et dans certains cas de suivre à distance les cours du bétail. L'internet donne une visibilité et une accessibilité internationale aux grandes organisations et réseaux d'éleveurs qui mettent en place des sites web.

\section{3 / Comment les éleveurs innovent-ils face aux chan- gements?}

Face aux opportunités et aux contraintes générées par ces changements, les éleveurs cherchent à faire évoluer leurs pratiques en innovant. 


\section{1 / Opportunités et contraintes liées aux changements}

Certains changements rendent nécessaire une évolution des pratiques actuelles. Concernant la mobilité des troupeaux dans les territoires et la gestion des pâturages, la réduction des parcours au profit des cultures et la fragmentation des territoires villageois limitent la mobilité des troupeaux. L'augmentation de la compétition sur les ressources fourragères fait peser des risques de dégradations irréversibles des écosystèmes. Le réchauffement climatique bouleverse le régime des pluies et rend la production primaire de biomasses et les réserves d'eau de saison sèche plus aléatoires. Concernant l'élévation de la productivité et l'amélioration de la rentabilité, la production est face à de nouvelles contraintes. La transformation des milieux et des systèmes d'élevage induisent de nouvelles pathologies animales (recul des trypanosomoses, apparition de nouvelles tiques). L'érosion génétique pourrait conduire à la disparition de génotypes locaux intéressants pour les milieux difficiles. La hausse du prix des matières premières entraîne une augmentation conséquente du prix des intrants (notamment aliments du bétail et engrais) et freine les stratégies d'intensification de la production. L'incidence accrue de phénomènes climatiques extrêmes (inondations, cyclones tropicaux, sécheresse) conduit à une plus grande variabilité de la production et à l'augmentation de la volatilité des prix. Concernant l'évolution de la demande, les filières de l'élevage accusent plus de retard que les filières agricoles. La qualité sanitaire des produits locaux pourrait souvent être améliorée pour satisfaire une demande de plus en plus exigeante sur cet aspect.

Mais certains changements procurent de nouvelles opportunités pour l'élevage. Ainsi, la croissance soutenue de la population et l'apparition de nouveaux modes de consommation induits par l'émergence d'une classe moyenne stimulent la demande en produit animaux dont on prévoit le doublement d'ici 2050. Lorsque que le niveau de pression foncière s'élève, et quand les prix des aliments flambent, alors on voit apparaître les prémices d'une intensification fourragère pour maintenir l'élevage dans les territoires (encadré 2). La structuration des organisations des éleveurs permet d'augmenter et d'améliorer l'offre locale et de distribuer plus équitablement la valeur ajoutée le long des filières. La diffusion des technologies de la communication et l'amélioration des infrastructures renforce aussi leur intégration au marché. La mise en place des collectivités locales chargées de la gestion des ressources naturelles permet aux éleveurs

Encadré 2. En attendant la révolution fourragère.

De l'après-guerre au milieu des années 1990, la recherche a essayé d'introduire les cultures fourragères pour faire face à la pénurie de ressource alimentaire en saison sèche (Roberge et Toutain 1999). Légumineuses et graminées furent proposées en culture pure, parfois en association ou sous forme de banques fourragères pour améliorer les jachères, fournir des aliments au bétail, restaurer les terres dégradées ou servir de plantes de couverture. Mais la " révolution fourragère " longtemps attendue est restée timide. Les producteurs considéraient qu'il n'y avait pas de main-d'œuvre disponible pour ces cultures, et que les ressources disponibles permettaient globalement de couvrir les besoins alimentaires des animaux moyennant une conduite extensive et une gestion des variations de leur état corporel. Peu à peu les acquis de la recherche et du développement se sont déstructurés (dispersion des banques de semences, arrêt de la vulgarisation...). Mais aujourd'hui, la donne change avec l'extension des cultures et la réduction des pâturages, les problèmes d'alimentation se posent dès la saison des pluies. Les éleveurs ne peuvent plus compter ni sur cette période ni sur les espaces de retrait en saison sèche (bas-fonds occupés par des cultures de contre saison), pour reconstituer les réserves corporelles de leurs animaux. Produire une biomasse fourragère de qualité devient un enjeu important et, dans les zones densément peuplées, on perçoit les prémisses d'une intensification fourragère (stockage des résidus agricoles, légumineuses à fins multiples).

de s'impliquer dans la gouvernance locale et de sécuriser l'accès aux ressources.

Face à ces nouvelles opportunités et contraintes, les éleveurs et les acteurs du secteur de l'élevage innovent. Comparativement à d'autres économies émergentes d'Asie et d'Amérique du Sud, ces innovations sont moins visibles. Mais des tendances se dessinent en fonction des contraintes et opportunités perçues localement et orientent les transformations de l'élevage. Pour illustrer ces transformations, nous avons choisi de présenter une sélection d'innovations liées à la levée de contraintes importantes comme la réduction de la mobilité du bétail et la réduction des pâturages, la faible productivité ou encore le manque d'intégration au marché.

\section{2 / Résoudre les problèmes d'ac- cès à la ressource et de mobilité des troupeaux}

Pour faire face aux problèmes d'accès à la ressource et de mobilité dans les territoires, nous présenterons deux situations où les collectivités et communautés locales s'organisent pour créer de nouveaux modes de gouvernance des territoires, en s'appuyant sur des politiques publiques innovantes (décentralisation en Afrique de l'Ouest, cogestion des communs au Maghreb).

a) Mise en place d'une charte foncière locale dans une commune rurale de l'Ouest du Burkina Faso

En Afrique de l'Ouest, les communes rurales récemment installées grâce à la décentralisation offrent de nouvelles opportunités pour la création de modes de gouvernance territoriale innovants. Ces innovations organisationnelles visent à mieux gérer la montée des compétitions et des conflits liés à l'usage des ressources agro-sylvo-pastorales et ainsi pallier aux insuffisances des autorités coutumières lorsque la pression foncière devient trop forte. Au Burkina Faso, la commune de Koumbia avait inscrit la réalisation d'une charte foncière locale dans son plan de développement et il s'agissait de voir comment mettre en place un tel dispositif en suivant le cadre juridique proposé par l'Etat burkinabé (Diallo et Vall 2012). La commune, la recherche (Cirdes, Cirad) et un cabinet de juriste ont travaillé ensemble à l'élaboration d'une charte de 2008 à 2012, dans le cadre du projet « Fertipartenaires » (Food/2007/144-075), en suivant un processus participatif comprenant trois phases (exploration, rédaction et application). Durant la phase exploratoire (2008-2009), un état des lieux, ainsi que des formations et un voyage d'étude, ont été réalisés en impliquant les 14 villages de la commune dans des cadres de concertation transitoires pour recenser les problèmes et identifier des propositions de règles. Durant la phase de rédaction (2010), une commission composée de représentants des acteurs de la commune a rédigé un projet de charte qui, suite à un long processus de validation dans les 14 villages et par les institutions de tutelles, a été adopté par le conseil municipal. En se référant aux us et coutumes locales, tout en veillant au respect des lois en vigueur au Burkina Faso, les chapitres de la charte traitent des règles d'accès à la terre, de gestion des espaces et ressources agricoles, pastoraux, forestiers et fauniques. Pour son application (2011-2012), des commissions villageoises foncières et de conciliation ont été mises en place et leurs membres ont été formés. En rénovant les règles d'accès au foncier et aux ressources, la charte offre aux éleveurs des conditions favorables pour garantir la mobilité des troupeaux, pour accéder aux pâturages et aux points d'eau et elle propose des recours en cas de problèmes. Mais s'il est relativement aisé de construire de 
nouvelles règles en s'appuyant sur un cadre juridique, il est difficile de faire changer les anciennes pratiques. L'application d'une telle charte repose fortement sur l'opérationnalité des commissions instituées.

b) Des dispositifs institutionnels de cogestion des parcours situés sur les communs au Maroc

Aujourd'hui, au Maghreb, la cogestion des parcours basée sur des régimes de propriété commune, alliant la décentralisation de la gestion des ressources et la participation des communautés, se développe. Elle s'appuie sur des réformes institutionnelles. Mais sa réussite est conditionnée par la délimitation de frontières communautaires pour que les bénéfices des efforts entrepris ne soient pas dispersés et accaparés par des membres extérieurs aux communautés. Cette délimitation ne doit pas se faire à l'encontre de la mobilité des troupeaux, essentielle dans ces milieux arides. Dans ce contexte, une étude (Icarda, Cirad, Inra Maroc) a été entreprise pour comprendre comment l'espace pastoral de la tribu des Béni Guil du Nord de la région d'Oujda (Est Maroc) composée de 9 fractions de Tendrara et Maatarka, est approprié et exploité par les différents groupes en présence et pour évaluer la faisabilité de l'approche de cogestion (Dutilly et al 2007). L'étude a montré que chaque fraction possédait des parcours propres avec néanmoins des frontières floues, que les proportions des terres appropriées variables d'un groupe à un autre traduisaient des rapports de dominance territoriale, et que les parcours appropriés par une fraction étaient moins dégradés avec des charges animales inférieures à celles des parcours collectifs. Parallèlement, certaines fractions avaient fortement recours à la mise en culture pour définir leurs territoires de pâturage. Les parcours semblaient se caractériser par des frontières floues entre fractions de la même tribu mais en revanche bien définies avec les groupes extérieurs, et pourraient donc se définir comme des parcours en propriété collective. L'organisation sociale de la gestion des parcours doit donc être comprise à une échelle très fine pour repérer les acteurs légitimes pour les négociations nécessaires à la mise en place d'une cogestion des parcours. Des dispositifs de négociation multiacteurs (associant acteurs de la recherche, du développement et de la société civile), intégrant les multiples fonctions sociales, économiques et environnementales assurées par ces zones de parcours, pourraient être utilisés pour faciliter les négociations locales, mais aussi nationales et régionales. Mais ces dispositions ne résolvent pas tous les problèmes, notamment ceux relatifs au maintien des populations dans des zones marginales, et ceux relatifs à l'augmentation des revenus des éleveurs qui pourraient appeler d'autres innovations comme la labélisation des viandes issues de parcours.

\section{3 / Augmenter la productivité des systèmes d'élevage}

Pour accroitre la productivité des élevages, nous présenterons deux situations d'innovations où l'objectif est atteint sans intensification importante en intrants (association de l'agriculture et de l'élevage à Madagascar), ou en capital (introduction du cactus inerme au Maghreb).

a) Améliorer la conservation des nutriments dans les exploitations de polycultureélevage à Madagascar pour accroître la productivité dans un contexte de pénurie d'intrants

Dans les Hautes Terres centrales de Madagascar les espaces disponibles sont de plus en plus rares (100 hab./ $\left.\mathrm{km}^{2}\right)$. Les jachères sont raccourcies et la fertilité des sols décroît. L'accès aux intrants (engrais, aliments, bétail) est très difficile en raison du contexte politico-économique. L'intensification de la production est nécessaire mais difficile à mettre en œuvre. Les petites exploitations de polyculture élevage étant largement majoritaires $(0,12$ à 2 ha, riz, légumes, 1 à 2 bœufs de trait, 1 à 4 vaches laitières, volaille, pisciculture...), l'association agriculture-élevage est privilégiée par les producteurs pour produire des effets d'intensification (via l'amélioration du recyclage des résidus de culture et des effluents d'élevage sur l'exploitation) tout en contribuant à restaurer la fertilité des sols. La recherche et le développement (Fifamanor, Fofifa, Essa, Cirad) conduisent des travaux pour évaluer l'efficience de la conservation des nutriments dans les exploitations, et concevoir des techniques innovantes associant les savoirs locaux et des avancées scientifiques concernant la gestion des effluents organiques (Salgado et Tillard 2012, Alvarez et al 2013). La conservation de l'azote est évaluée au long du processus de transfert de l'animal (fèces, urines) à la plante (fixation) et au sol (minéralisation) grâce à des suivis de pratiques et à la spectrométrie dans le proche infrarouge. Les effluents d'élevage, les résidus agricoles et la végétation naturelle, autrefois délaissés ou brûlés, sont désormais gérés avec parcimonie. L'association agriculture-élevage est très développée pour les travaux culturaux et les transports (énergie animale), pour la production de fertilisants organiques (fumier, poudrette, compost pour le riz et les cultures maraichères), pour l'alimentation des animaux (foin naturel fauché, cultures fourragères de légumineuses et céréales et divers sous-produits agricoles). L'utilisation d'une dalle sur le sol des étables, l'ajout de paille de riz dans la litière, le stockage du fumier en fosse, l'ajout de lisier de porc ou de volaille au fumier et la réduction du temps de stockage améliorent significativement la teneur en azote des fumiers $(<20$ gN/kgMS en pratique traditionnelle contre $260 \mathrm{gN} / \mathrm{kgMS}$ en pratique améliorée) et l'efficience de la conservation de l'azote $(<15 \%$ en pratique traditionnelle contre $60 \%$ en pratique améliorée). Les recherches se poursuivent pour évaluer l'effet de ces innovations sur le relèvement de la fertilité du sol, l'amélioration de l'autosuffisance alimentaire, l'augmentation des revenus des familles et l'efficience environnementale de ce type d'agriculture.

b) L'introduction du cactus inerme au Maghreb induisant des innovations organisationnelles et des gains de productivité sans intensification en capital

Au Maghreb, 45\% des terres sont exposées à la désertification en raison du surpâturage, de la mécanisation, de la déforestation, des pollutions du sol et des stress hydriques et thermiques importants. L'extension de la céréaliculture, l'augmentation du cheptel et la déstructuration des organisations traditionnelles de gestion des ressources collectives ont participé à l'accélération de la désertification. Le modèle d'intensification des années 1970-80 est remis en cause. Des innovations techniques et sociales sont nécessaires pour maintenir l'économie pastorale ou agro-pastorale (Alary 2003, Alary et al 2007). Ce fût l'objectif de l'Icarda dans le Mashreq/Maghreb Project. Dans ce dernier, on mit d'abord l'accent sur des technologies adaptées aux conditions socio-économiques et naturelles au moyen d'une approche participative (1995-1998). Puis, en appliquant un modèle communautaire de diffusion de l'innovation, on s'est intéressé aux formes d'organisation susceptibles de soutenir l'adoption de l'innovation, et des équipes multidisciplinaires et multi-institutionnelles ont développé des options intégrées technologiques, institutionnelles et politiques. Le Cirad a été associé à l'évaluation des impacts économiques, sociaux et environnementaux des technologies développées. L'introduction du cactus inerme pour l'alimentation animale a connu un réel succès mais principalement sur les terres privatives. Son succès tient au fait qu'il a été adopté sans peser sur les budgets ou sur le temps de travail des ménages (récolte, transport, découpe), et ce malgré une valeur alimentaire assez faible. En revanche, les innovations plus intensives comme les semences améliorées d'orge, le bétail amélioré, l'introduction de blocs alimentaires, n'ont pas eu le même succès, car elles étaient trop dépendantes 
du marché ou des politiques publiques. Néanmoins, le développement du cactus a montré comment il peut y avoir des synergies positives entre politiques agropastorales et accompagnement social, entre savoir local et savoir scientifique, mais aussi comment une technologie sécurisante peut participer à l'augmentation de la productivité sans intensification en capital. L'analyse des mécanismes de négociations sociales dans le processus de diffusion de l'innovation a montré enfin que le soutien social, voire moral, assuré par les agents du développement et les chercheurs a eu un rôle important. Dès lors, on voit comment la technologie n'est qu'une composante de l'innovation et comment les chercheurs doivent répondre aux attentes et s'inscrire dans les logiques de reproduction des sociétés dans lesquelles le savoir scientifique se construit.

\section{4 / Répondre à la demande urbaine en produits animaux}

Nous prendrons l'exemple de l'industrie laitière en Afrique de l'Ouest, où certaines laiteries substituent de plus en plus la production de lait locale aux importations, évoluant ainsi vers un modèle mixte de laiteries. Dans cette région l'approvisionnement des villes en produits laitiers locaux est toujours insuffisante (à Dakar et Bamako, $90 \%$ de la demande est couverte par de la poudre de lait importée). La croissance urbaine éloigne toujours plus les bassins de production, renchérissant les coûts de transport. Des industries laitières poursuivent leur développement sur la base d'importations de lait en poudre. Les bassins laitiers les moins bien connectés au marché subissent davantage les effets des politiques de libéralisation (Duteurtre 2009, Corniaux et al 2012a).

Pour comprendre l'impact de ces politiques sur la localisation des élevages et sur les dynamiques de développement territorial des recherches sont conduites sur des bassins laitiers périurbains au Sénégal, en Mauritanie, au Niger et au Mali (Duteurtre 2007, Corniaux et al 2012 b). Défendre la production locale face à l'ouverture des marchés, passe aujourd'hui par le développement de circuits mixtes utilisant à la fois le lait en poudre et le lait local. Les expériences de la Laiterie du Berger (Sénégal), de Tiviski (Mauritanie), de Niger-Lait (Niger) et de Mali-Lait (Mali), montrent qu'aujourd'hui ces circuits mixtes sont rentables. Pendant longtemps ce ne fût pas le cas. La poudre de lait entrait sur le marché africain à un prix inférieur à celui de la production locale parce que l'Europe écoulait sa surproduction sur le marché mondial à un prix subventionné et parce que les Etats africains taxaient faiblement ce type d'importation. Désormais la donne a changé. Les cours mondiaux du lait sont remontés, l'Europe abandonne sa politique de quotas et réduit les subventions à l'export et en Afrique les taxahausse pour les produits laitiers transformés. La production locale est devenue plus attractive pour les industriels. Sur les marchés de niche typés et rémunérateurs des produits laitiers gras (fromage sans mélange. Sur le marché de masse concurrentiel du lait frais et du lait caillé, elle est valorisée avec mélanges, afin de proposer un produit goûteux recherché par les consommateurs. Cette nouvelle dynamique de l'industrie laitière a été impulsée par un faisceau de changements propres au secteur laitier (volatilité du cours mondial de la poudre de lait, diversification de la gamme des produits laitiers), structurels (amélioration des transports, électrification, formation et qualification de la main-d'œuvre) et sociétaux (nouveaux modes de consommation des urbains).

\section{4 / Evolutions de l'élevage induites par les changements et facteurs de changements}

\section{1 / Evolutions des systèmes d'éle- vage induites par les changements}

Aujourd'hui, les évolutions des systèmes d'élevage résultent de deux processus concomitants et dépendants.

Il y a, d'une part, les transformations liées aux innovations qui se caractérisent par une certaine lenteur et discrétion, la recherche du " moindre coût » (énergétique, financier, social ; cf. Vall et al 2012 pour le cas de l'association agriculturetions à l'importation sont revues à la blanc, crème fraiche) elle est valorisée

élevage) parce que les incertitudes sont grandes et les filets de sécurité inexistants quand on se situe aux marges de l'économie monde.

Il y a, d'autre part, les processus de croissance plus rapides et visibles sur le terrain, comme l'augmentation du cheptel, la diffusion rapide et généralisée des systèmes mixtes de polyculture-élevage, et, dans une moindre mesure parce que plus localisée, l'éclosion des systèmes intensifs et spécialisés.

Désormais, le niveau de dépendance directe aux ressources naturelles et le niveau d'intégration au marché (des biens, des intrants, des services et du travail) constituent deux facteurs de différentiation majeurs des systèmes d'élevage (figure 3 ).

Avec l'augmentation de la pression foncière et la réduction des espaces pastoraux, la majorité des agro-pasteurs mixtes et une part non négligeable des pasteurs entrent aujourd'hui dans une logique d'intensification de la production, modérée par l'augmentation du prix des intrants et les nombreuses incertitudes liées aux aléas climatiques et à la volatilité des prix agricoles. On pourrait la qualifier d'intensification à moindre coût financier, représentée notamment par la pratique de l'intégration de l'agriculture et de l'élevage. Ces incertitudes conduisent aussi les éleveurs à diversifier les productions animales et végétales, à développer des activités extra-agricoles, à effectuer des migrations temporaires ou définitives pour une partie des membres de l'exploitation quand cela est possible. Dans les années à venir les systèmes mixtes agro-pastoraux vont prendre encore plus d'importance, en réduisant leur dépendance directe aux

Figure 3. Schématisation de la diversité, du nombre et des évolutions possibles des systèmes d'élevage en Afrique en lien avec les changements.

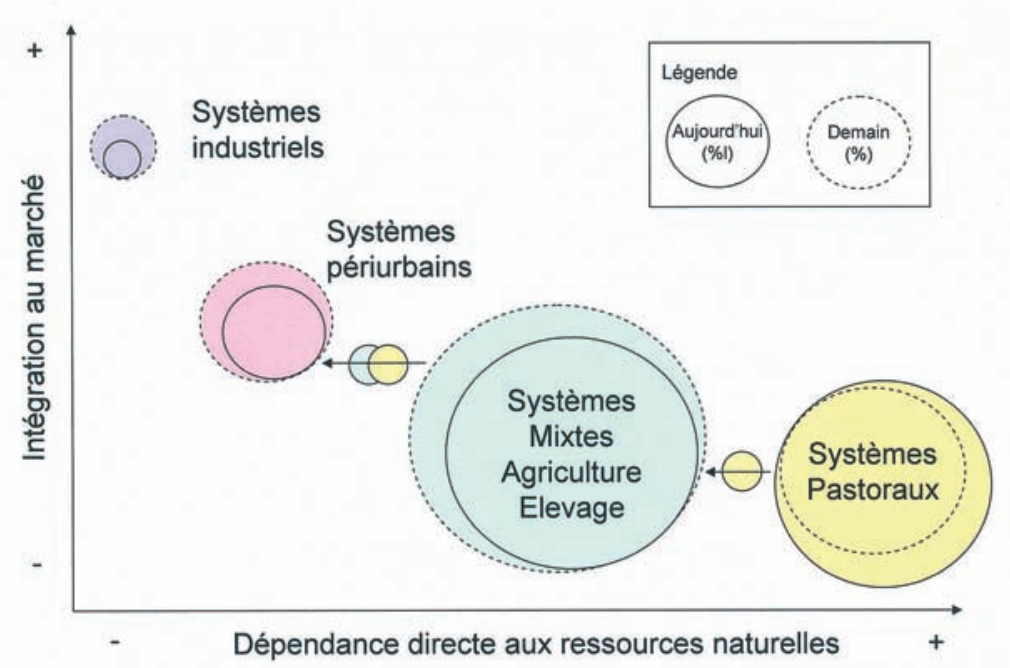


ressources naturelles et en renforçant leur intégration au marché. Dans les zones pastorales une fraction des élevages pourrait aussi évoluer vers des systèmes mixtes quand les conditions locales le permettent (disponibilité en terre agricole et accès à un marché).

Aussi, l'augmentation de la population, la forte croissance urbaine, l'émergence d'une classe moyenne se conjuguent pour stimuler la demande en produits animaux en quantité, en qualité et en diversité. Néanmoins une pression élevée sur les prix persiste, d'une part en raison des revenus limités de la majorité des ménages et, d'autre part, à cause de la concurrence des importations extra-africaines. On peut donc avancer que les élevages qui entreront dans des logiques de spécialisation de la production et d'économie d'échelle pour mettre sur le marché des produits à bas prix, seront les mieux positionnés pour répondre à ce type de demande sociétale. Ainsi, les élevages périurbains familiaux et semi-industriels devraient connaître un développement important dans les années à venir. Mais la spéculation foncière à l'œuvre depuis quelques années dans la périphérie des grandes mégapoles pousse de nombreux éleveurs à vendre leur lopin de terre pour aller s'installer en périphérie agricole. Quant aux élevages industriels, leur développement est aussi conditionné par une capacité d'investissement suffisante, l'existence de circuits de distribution bien structurés, la présence de filières organisées et d'un environnement des affaires sécurisant ce qui, compte tenu de la situation générale de la majorité des pays africains, freine pour l'instant leur développement.

\section{2 / Conséquences en retour des évolutions sur les changements}

Plus lentes et progressives, les transformations des systèmes d'élevage liées aux innovations, favorisent la maîtrise du changement, notamment parce qu'elles procèdent par ajustements successifs et par tissage de réseaux d'acteurs (et des contrats afférents écrits ou tacites...), alimentés par des débats et des jeux d'acteurs (illustrés dans cet article par la mise en place de dispositifs de gouver- nance et de cogestion territoriale, par la construction de modèles de transformations du lait, par de nouvelles formes d'agencements des composantes des exploitations...). Concernant ce premier type d'évolution de l'élevage, chercher à l'accélérer sans perdre la maîtrise des choses pour accroître la production tout en respectant l'environnement, constitue un enjeu de recherche et de développement très important. Ce premier enjeu milite en faveur du développement de travaux sur la co-conception pas à pas de systèmes d'élevage innovants.

Plus massifs et rapides, les processus de croissance et de réplication plus ou moins à l'identique des modèles d'élevage actuels sans remise en question des pratiques, risquent d'aggraver non seulement les changements en cours, mais aussi les effets en retour des changements sur les élevages. Ainsi, la poursuite des logiques d'extension des troupeaux et de conduites extensives que l'on voit souvent à l'œuvre risque d'aggraver la dégradation des milieux, de stimuler la montée des conflits entre agriculteurs et éleveurs, de potentialiser les effets du changement climatique, sans améliorer substantiellement les capacités de la production locale à satisfaire l'évolution de la demande. Par rapport à ce second type d'évolution, amener les acteurs de l'élevage à réfléchir aux conséquences des évolutions actuelles sans changements de pratiques, à imaginer des modes de production innovants éventuellement en rupture avec la situation présente, à proposer des mesures innovantes (des réformes institutionnelles et des politiques publiques innovantes et cohérentes permettant de créer des conditions favorables à l'accroissement de la productivité et de la durabilité), dans le cadre d'exercices de conceptions de systèmes d'élevage de novo constitue un autre enjeu de recherche et de développement.

\section{Conclusion et perspectives}

En Afrique, de multiples changements ont affecté l'environnement local et global des systèmes d'élevage au cours des 50 dernières années. Certains changements ont été source de fragilisation, d'autres porteurs d'opportunités. Ils ont induit des transformations et aujourd'hui des tendances se dessinent. Dans les systèmes pastoraux l'innovation concerne principalement la gestion des ressources pastorales et la mobilité du bétail, dans les systèmes agro-pastoraux principalement la diversification et l'intégration agriculture-élevage, et dans les systèmes périurbains et industriels principalement la spécialisation et l'intensification de la production par les intrants, le capital et le travail. De plus en plus de producteurs s'orientent vers des systèmes mixtes de polyculture-élevage, le pastoralisme se maintient et permet de valoriser d'immenses territoires le plus souvent arides, et dans les zones périurbaines une forte demande en produits d'élevage entraîne la mise en place de systèmes d'élevage plus intensifs et plus spécialisés.

Aujourd'hui les enjeux à venir de l'élevage en Afrique se posent dans un contexte de volatilité extrême des prix des intrants, de paupérisation de larges franges de la population, de réduction des espaces disponibles pour l'élevage, de crise du pastoralisme et d'émergence des questions environnementales dans les évaluations des performances des productions animales. Les perspectives offertes par les réalités actuelles de l'élevage africain, notamment sa faible productivité et la demande accrue en produits animaux attendue d'ici à 2050 devraient lui assurer un marché et des marges de progression significatives. Toutefois, ceci ne pourra se réaliser que moyennant $i$ ) la poursuite des réformes institutionnelles notamment celles concernant la sécurisation du foncier, l'accès aux services et aux intrants, $i i$ ) une implication directe des organisations de producteurs concernant l'entrée des produits locaux sur le marché, le maintien de l'élevage dans les territoires, le renforcement des capacités techniques (cultures fourragères, amélioration génétique) et de gestion des éleveurs, iii) des termes du marché plus sécurisés notamment face aux importations des produits animaux subventionnés et $i v)$ l'amélioration de l'appui technique concernant le gestion de l'élevage, la reconnaissance des savoirs locaux et l'adaptation des connaissances scientifiques au contexte local.

\section{Références}

Abdelguerfi A., Laouar M., 2000. Conséquences des changements sur les ressources génétiques du Maghreb. In : Rupture : nouveaux enjeux, nouvelles fonctions, nouvelle image de l'élevage sur parcours. Bourbouze A., Qarro M. (Eds). CIHEAM, Montpellier, France, 77-87.

Alary V., 2003. Des processus d'adoption de l'in-novation dans les zones vulnérables Exem-ple d'un projet de recherche et dévelop- pement au Maghreb. Revue Afrique Contemporaine, 219, 81-101.

Alary V., Lhoste P., 2002. Le diagnostic des systèmes d'élevage. In : Mémento de 1'Agronome. MAE, CIRAD, GRET, Paris, France, 1239-1266.

Alary V., Nefzaoui A., Ben Jemaa M., 2007. Conditions of development of a technology in arid and semi-arid areas. Modeling impact of spineless cactus in alley cropping in Central Tunisia. Agricult. Sys., 94, 573-585.

Alary V., Duteurtre G., Faye B., 2011. Elevage et sociétés : les rôles multiples de l'élevage dans les pays tropicaux. In : Numéro spécial, Elevage en régions chaudes. Coulon J.B., Lecomte P., Boval M., Perez J.M. (Eds), INRA Prod. Anim., 24, 145-156. 
Alvarez S., Rufino M.C., Vayssières J., Salgado P., Tittonell P., Tillard E., Bocquier F., 2013. Whole-farm nitrogen cycling and intensification of crop-livestock systems in the highlands of Madagascar: an application of network analysis. Agricult. Sys. (DOI 10.1016/j.agsy.2013.0 3.005).

Bichat H., 2012. Et si l'agriculture sauvait l'Afrique? Quae Editions, Paris, France, 159p.

Bourbouze A., 2007. Regards sur les pastoralismes du monde et du nord de l'Afrique. Entre résistance et mutations. Réseau méditerranéen Pastoralisme et société. https://resopasto. iamm.fr/index.php/fr/

Boutonnet J.P., Griffon M., Viallet D., 2001. Compétitivité des productions animales en Afrique subsaharienne et à Madagascar : synthèse générale. MAE, Ministère de la coopération et du développement, Paris, France, 191p.

Bouwman A.F., Van der Hoeck K.W., 1997. Scenarios of animal waste production and fertilizer use and associated ammonia emission for the developing countries. Atmosph. Environ., 31, 4095-4102.

Club du sahel, 2008. Elevage et marché régional au Sahel et en Afrique de l'Ouest. Potentialités et défis. CSAO-OCDE, CDAO, Paris, France, $182 \mathrm{p}$.

Corniaux C., Alary V., Gautier D., Duteurtre G., 2012a. Producteur laitier en Afrique de l'Ouest : une modernité rêvée par les techniciens à l'épreuve du terrain. Autrepart, 62, 17-36.

Corniaux C., Vatin F., Ancey V., 2012b. Lait en poudre importé versus production locale en Afrique de l'Ouest : vers un nouveau modèle industriel ? Cah. Agric., 20, 18-24.

Coulon J.B., Lecomte P., Boval M., Perez J.M., 2011. Numéro spécial, Elevage en régions chaudes. INRA Prod. Anim., 24, 160p.

Daburon, A., 2013. Urban and Peri-urban Milk Producers of El Cairo City: an Efficiency Focus. Master Ecologie-Biodiversité, Université MontpellierII/CIRAD, 73p.

Dedieu B., Aubin J., Duteurtre G., Alexandre G., Vayssières J., Bommel P., Faye B., 2011. Conception et évaluation de systèmes d'élevage durables en régions chaudes. In : Numéro spécial, Elevage en régions chaudes. Coulon J.B., Lecomte P., Boval M., Perez J.M. (Eds), INRA Prod. Anim., 24, 113-128.

Diallo M.A., Vall E., 2012. La construction d'un dispositif de gouvernance territoriale pour la gestion des ressources naturelles : le cas de la charte foncière locale de Koumbia. In : Partenariat, modélisation, expérimentations : quelles leçons pour la conception de l'innovation et l'intensification écologique ? Vall E., Andrieu N., Chia E., Nacro H.B. (Eds). Séminaire DP Asap, Bobo Dioulasso, Burkina Faso, http://hal.archives-ouvertes.fr/ASAP

Dongmo A.L., Djamen P., Vall E., Koussou M.O., Coulibaly D., Lossouarn J., 2007. L'espace est fini! Vive la sédentarisation ? Innovations et développement durable en question chez les pasteurs des zones cotonnières d'Afrique de l'Ouest et du Centre. In : Renc., Rech., Rum., 14, 153-160.
Dorin B., Paillard S., Treyer S., 2011. Agrimonde. Scenarios and challenges for feeding the world in 2050. Quae Editions, Versailles, France, 296p.

Duteurtre G., 2007. Commerce et développement de la filière laitière en Afrique de l'Ouest : une synthèse. Revue Elev. Méd. Vét. Pays Trop., 60, 209-223.

Duteurtre G., 2009. Lait des pauvres, lait des riches : réflexion sur l'inégalité des règles du commerce international. In : L'élevage, richesse des pauvres. Duteurtre G., Faye B. (Coord). Quae Editions, Paris, France, 249-266.

Dutilly C., Acherkouk M., Bechchari A., Bouayad A., El Koudrim M., Maatougui A., 2007. Dominance communautaire dans l'exploitation des espaces pastoraux : impacts sur les modes de vie et implications pour la gestion des parcours du Maroc oriental. Cah. Agric., $16,338-346$.

FAO, 2009. La situation mondiale de l'alimentation et de l'agriculture. Le point sur l'élevage. FAO, Rome, Italie, 202p.

Faure G., Havard M., Toillier A., Djamen Nana P., Moumouni I., 2014. Innovations dans les services de conseil aux exploitations agricoles familiales. In : Agricultures familiales et mondes à venir. Sourrisseau J.M. (Ed), Quae Editions, Paris, 257-268.

Faye B., Alary V., 2001. Les enjeux des productions animales dans les pays du Sud. INRA Prod. Anim., 14, 3-13.

GIEC, 1996. Lignes directrices du GIEC pour les inventaires nationaux de gaz à effet de serre, version révisée 1996, Reference Manual (Volume 3). GIEC, Genève, Suisse, 483p.

Herrero M., Thornton P.K., Notenbaert A.M., Wood S., Msangi S., Freeman H.A., Bossio D., Dixon J., Peters M., van de Steeg J., Lynam J., Parthasarathy Rao P., Macmillan S., Gerard B., McDermott J., Séré C., Rosegrant M., 2010. Smart investments in sustainable food production: revisiting mixed crop-livestock systems. Science, 327, 822-825.

IIED, 2009. Modern and mobile. The future of livestock production in Africa's drylands. IIED and SOS Sahel, London, UK, 88p.

IPCC, 2007. Africa: Climate change 2007. In: Impacts, Adaptation and Vulnerability, Contribution of Working Group II (Chapter 9), Cambridge University Press, UK, 433-469.

Lancelot R., Zundel E., Ducrot C., 2011. Spécificité de la santé animale en région chaude : le cas des maladies infectieuses majeures en Afrique. In : Numéro spécial, Elevage en régions chaudes. Coulon J.B., Lecomte P., Boval M., Perez J.M. (Eds). INRA Prod. Anim., 24, 65-76.

Lhoste P., Havard M., Vall E., 2010. La traction animale. Quae, CTA, PAG, Paris, France, 224p.

Meynard J.M., Dedieu B., Bos A.P., 2012. Re-design and co-design of farming systems. An overview of methods and practices. In: Farming systems Research into the 2\&st century : the new dynamic. Darnhofer I., Gibon D., Dedieu B. (Eds.). Springer, 407-432.
Petit M., 2011. Pour une agriculture productive et durable. Quae Editions, Paris, France, 112p.

Planchenault D., Boutonnet J.P., 1997. Conservation de la diversité des ressources génétiques animales dans les pays d'Afrique francophone sub-saharienne. Anim. Genet. Res. Inf., 21, 1-22.

Poccard-Chappuis R., Bonaudo T., Tourrand J.F., Lossouarn J., 2011. Elevage, filières et territoires en régions chaudes. In: Numéro spécial, Elevage en régions chaudes. Coulon J.B., Lecomte P., Boval M., Perez J.M. (Eds). INRA Prod. Anim., 24, 129-144.

Roberge G., Toutain B., 1999. Cultures fourragères tropicales. Collection Repères, Cirad, Quae Editions, Paris, France, 369p.

Robinson T.P., Thornton P.K., Franceschini G., Kruska R.L., Chiozza F., Notenbaert A., Cecchi G., Herrero M., Epprecht M., Fritz S., You L., Conchedda G., See L., 2011. Global livestock production systems. FAO, ILRI, Rome, Italy, 152p.

Salgado P., Tillard E., 2012. Conservation des ressources fertilisantes dans les systèmes d'élevage des pays du Sud. Salon International de l'Agriculture de Paris 2012. Fiche de compétences, Cirad, Montpellier, France, 2p.

Sraïri M.T., 2011. Développement de l'élevage au Maroc : succès relatifs et dépendance alimentaire. Courrier de l'Environnement de I'INRA, 60, 91-101.

Steinfeld H., Gerber P., Wassemar T., Castel V., Rosales M., De Haan C., 2006. Livestock's long shadow. Environmental issues and options. FAO, Rome, Italy, 390p.

United Nations, 2010. World Population Prospects: The 2010 Revision, Population Database, United Nations Population Division, New York (http://esa.un.org/unpd/wpp/index.htm)

Vall E., Chia E., 2014. Co-construire l'innovation : la recherche-action en partenariat. In : Agricultures familiales et mondes à venir. Sourrisseau J.M. (Ed). Quae Editions Paris, 239-256.

Vall E., Diallo M.A., 2009. Savoirs techniques locaux et pratiques: la conduite des troupeaux aux pâturages (Ouest du Burkina Faso). Natures Sci. Soc., 17, 122-135.

Vall E., Lhoste P., Abakar O., Dongmo Ngoutsop AL., 2003. La traction animale dans le contexte en mutation de 1'Afrique subsaharienne: enjeux de développement et de recherche. Cah. Agric., 12, 219-26.

Vall E., Blanchard M., Diallo M.A., Lecomte P., 2012. L'innovation par simplification expliquée par le principe de moindre quantité d'action de Maupertuis : cas de l'intégration agricultureélevage en Afrique soudano-sahélienne. Renc., Rech., Rum., 19, Paris, France, 401-404.

World Bank, 2008. World Development Report 2008. Agriculture for Development. Oxford University Press for the World Bank, Washington DC, USA, 386p. 


\title{
Résumé
}

En Afrique, les productions animales font face à une évolution importante de la demande et sont en proie à de multiples changements. Les systèmes d'élevage du futur devront être à la fois plus productifs et respectueux de l'environnement et pour cela les éleveurs devront innover. Aujourd'hui les systèmes agro-pastoraux deviennent majoritaires, le pastoralisme se maintient notamment dans les zones arides, et les élevages périurbains et spécialisés prennent de l'importance. Les changements locaux et globaux survenus au cours des 50 dernières années créent davantage de contraintes mais offrent de nouvelles opportunités. Des innovations sont à l'œuvre. En zone pastorale, elles concernent principalement la gestion des ressources et la mobilité du bétail, en zone agro-pastorale la diversification et l'intégration agriculture-élevage, en zone périurbaine la spécialisation et l'intensification de la production. Difficiles à déceler, lentes et progressives, les innovations favorisent la maîtrise du changement et doivent être encouragées et stimulées pour répondre aux enjeux de l'élevage. Les perspectives offertes par les réalités actuelles de l'élevage africain (faible productivité et progression rapide de la demande) devraient lui assurer un marché et des marges de progression significatives. Toutefois, ceci ne pourra se réaliser que moyennant la poursuite des réformes institutionnelles, une implication directe des organisations de producteurs, des termes du marché plus sécurisés et l'amélioration de l'appui technique.

\begin{abstract}
Changes and innovations in livestock systems in Africa

In Africa, animal production is facing a significant demand evolution and is marked by many changes. In the future, livestock systems should be more productive and environmentally friendly. In order to do so breeders have to innovate. Agro-pastoral systems occupy an increasingly important place, pastoralism continues especially in arid areas and peri-urban and specialized breeding are gaining importance. Local and global changes, over the past 50 years, create more constraints but offer new opportunities. Innovations are at work. In pastoral areas, they mainly concern the management of resources and mobility of livestock, in agro-pastoral area diversification and crop-livestock integration, and in peri-urban area specialization and intensification of production. Difficult to detect, slow and incremental, innovations promote the mastery of change and should be encouraged and stimulated to meet the challenges of animal husbandry. The opportunities offered by the current realities of African husbandry (low productivity and rapid growth in demand) should guarantee a market and significant margins of progression. However, this can be achieved through the continuation of institutional reforms, the direct involvement of producer organizations, the terms of the market more secure, improved technical support.
\end{abstract}

VALL E., SALGADO P., CORNIAUX C., BLANCHARD M., DUTILLY C., ALARY V., 2014. Changements et innovations dans les systèmes d'élevage en Afrique. In : Numéro spécial, Quelles innovations pour quels systèmes d'élevage? Ingrand S., Baumont R. (Eds). INRA Prod. Anim., 27, 161-174. 